\title{
A minimum weight labelling method for determination of a shortest route in a non-directed network
}

\author{
*Santosh Kumar \\ Department of Mathematics and Statistics, \\ University of Melbourne, Parkville, Melbourne, Australia \\ (skumar@ms.unimelb.edu.au) \\ Elias Munapo \\ Department of Decision Sciences, University of South Africa \\ Box 392 Unisa 0003, South Africa \\ (munape@unisa.ac.za) \\ Ozias Ncube \\ Graduate School of Business and Leadership \\ Box 392 Unisa 0003, South Africa \\ (ncubeo@unisa.ac.za) \\ Caston Sigauke \\ Department of Statistics and Operations Research \\ University of Limpopo \\ Bag X1106, \\ Sovenga 0727, South Africa \\ (csigauke@gmail.com) \\ Philimon Nyamugure \\ Department of Applied Mathematics \\ National University of Science and Technology \\ Box AC 939, Ascot, Bulawayo, Zimbabwe \\ (pnyamugure@gmail.com)
}

December 31, 2012

*Corresponding author: Tel.: ; Fax: ; Email address: skumar@ms.unimelb.edu.au 


\begin{abstract}
This paper proposes a new minimum weight labelling method for determining a shortest route in a non-directed network from a source node to a destination node. It is assumed that all links have positive weights associated with them. The key feature of this method is that for an $m$ node network the algorithm developed finds an optimal solution in at most $(m-1)$ iterations.
\end{abstract}

Key words: Non-directed network; shortest path; minimum weight algorithm.

\title{
1 Introduction
}

The shortest path problem deals with determination of a shortest path between two vertices of a graph such that sum of the weights of the constituent edges is minimized. Many industrial problems can be formulated and analyzed as a shortest path problem or its variants in a network. This has been of interest to researchers and practitioners in many network related disciplines like transportation, operations research and geographic information system technology (GIS), emergency services like supply of blood or reporting of ambulance at the accident scene. Many approaches have been developed to find the shortest path from a given node to all other nodes and appropriate computer codes have been developed for implementation of their algorithms. Many variants to this problem have been discussed in literature. Applications of shortest path also arise in many different areas, where a problem can be viewed as a network problem and therefore network methods can be applied to solve such problems. The problem of shortest route has been studied in directed as well as in non-directed networks (Ford, 1956; Dantzig, 1960; Dijkstra (1959); Floyd, 1962; Zhan, 1997; Zhan and Noon, 1998; among others). Many variants of the shortest path problem have been formulated and algorithms developed (Bansal and Kumar, 1977; Kumar et al., 1999; Munapo et al., 2008; among others).

In this paper we propose a new approach for determining a shortest path for a non-directed network. The rest of the paper is organized as follows. The minimum weight labelling method for the directed network is discussed in Section 2. Mathematical challenges created by the non-directed graph are presented in Section 3. Section 4 presents the minimum weight algorithm for the non-directed network developed in this paper. An illustrative example is given in Section 5 and in Section 6 we present some concluding comments. 


\section{Minimum weight label method for directed net- work}

The minimum weight label method for the directed network was developed by (Munapo et al., 2008). This method alters the weight associated with directed links entering a node by subtracting the minimum weight from all the incoming links and by adding the same minimum weight to the weights of all links departing from that node, thus leaving the total weight unaffected along any path from the source node to the destination node through that node as an intermediate node. In a directed network a link $(i, j)$ joins the nodes $i$ and $j$. The node $i$ indicates the start of the activity and the node $j$ indicates the end of that activity and it is further possible to assume without any loss of generality that $(i<j)$, i.e. the nodes of the network are to be numbered according to topological sort of the nodes. Let the source node be denoted by node 1 and the destination node be denoted by node $n$. All nodes of the network, other than the source node and the sink nodes, have at least one incoming and at least one outgoing link from that node. These intermediate nodes will be represented by $2,3, \ldots,(n-1)$. A label to a node assigns two values: a number $m$ representing its sequential position in the network as was assigned to it by the topological sort, and a weight $w$ representing the shortest distance from the source node to that node. These two numbers associated with a node form its label. The shortest distance from the source node to that node is denoted by $w$ is now known and also the path that leads to the shortest distance can also be easily traced out with the help of the label $m$. The minimum weight label algorithm, discused in Munapo et al. (2008), is made of the following steps:

Step 1: Initially label only the source node (denoted by the node 1) which is $(1,0)$ indicating that one arrives to node 1 from the node 1 and the total distance from node 1 to node 1 is 0 .

Step 2: Set $k=2$, and go to Step 3 .

Step 3: At node $k$, find the minimum weight, $w_{k}=\min \left\{w_{l 1, k} ; w_{l 2, k} ; \cdots ; w_{l k, k}\right\}$. Here it is assumed that at node $k$, there are $l_{k}$ number of incoming links. Using the minimum weight $w_{k}$, modify the associated weights with all incoming links by subtracting $w_{k}$ from their existing weights and by adding the same weight $w_{k}$ to the existing weights of the outgoing links from the node $k$. Go to Step 4.

Step 4: If $k<(n-1)$, set $k=k+1$ and return to Step 3 .

Step 5: All nodes have been labeled except the destination node $n$. It means the minimum weight $w_{n}$ associated with the destination node $n$ can be determined to conclude the shortest distance between the source and the destination node $n$. Note $w_{n}$ is the minimum weight associated with the incoming links with the destination node $n$. The corresponding path can also be traced by backtracking with the help of the first element of the label 
at node $n$.

\section{Shortest path in a non-directed network}

\subsection{Challenges in the case of a non-directed network}

In the case of a non-directed network, the above steps are no longer possible even if we convert the non-directed links into directed links as the very structure of the network undergoes unmanageable changes. For example, a non-directed link $(i, j)$ can be replaced by equivalent directed links $(i \rightarrow$ $j),(j \rightarrow l)$ and $(l \rightarrow i)$. Here node $l$ is an extra node and links $(j, l)$ and link $(l, i)$ are extra links. The weight associate with one of the extra links can be zero and weight with the other link can be the original weight associated with the link $(i, j)$. Thus each non-directed link conversion to directed link is achieved by introducing two additional links and one extra node. Thus the network $N(M, L)$, where $M$ is the node set and $L$ is the link set, will transform into a network $N^{\prime}(M+L, 3 L)$ network. This makes the transformed network unwieldy and in spite of that, it still is not going to satisfy the property that each directed link $(i \rightarrow j)$ will be such that the property $i<j$ will not be satisfied. Furthermore, non-directed connected networks are cyclic this aspect will further add difficulties.

Thus other properties of the non-directed network have to be exploited. These ideas are explained in the next subsection.

\subsection{Mathematical Background - Non-directed Network}

Let the given situation be formulated and represented as a non-directed network comprised of a node set $\{M\}$ and the link set $\{L\}$ and the network be denoted by $N(M, L)$. Let the cardinality of these two sets be denoted by $|M|$ and $|L|$ respectively. Let the origin node be denoted by $O$ and the destination node be denoted by $D$, then the number of other intermediate nodes in the network is given by $(|M|-2)$. It is assumed that the given situation is represented by a single-source, single destination non-directed network with non-negative link distances.

The following definitions are necessary for development of the algorithm.

\section{Definition 1. A node label}

A three element label is assigned to a node $k$ when the shortest route and the corresponding shortest distance from the origin node $O$ to that node is known. The three-element label at node $k$ is denoted by $k_{(i, j, d)}$, where the number $i$ indicates the order of labeling. Thus $i$ will have integer values $0,1,2, \ldots,(M-1)$. Alternatively $i$ also indicates the order of the distance

of that node from the origin node $O$. The number $j$ indicates the node on 
the path leading to the node $k$. This information helps to trace the shortest path leading to the specified cost. Finally the weight $d$ indicates the shortest distance from the origin node $O$ to the node $k$. Initially, only the origin node $O$ can be labeled as the distance from the node $O$ to itself is zero and the path is from node $O$ to itself means that travel has not been commenced. The value of $i$ is zero as it is the nearest node to the origin node $O$. Thus, initially, the label at the origin node $O$ will be $O_{(0, O, 0)}$ and all other nodes will be unlabeled. The third element of the label on a node indicates the total shortest distance between the origin node and that particular node, thus the value of the third element $d$ is also zero at the origin node.

\section{Definition 2. The sets of nodes and links}

$L_{0}=$ the set of labeled nodes. Initially the set $L_{0}=\{O\}$.

$L_{1}=$ Is the set of links that are directly joining labeled nodes to unlabeled nodes. Initially it will be comprised of links $(O, k)$, where a node $k$ is a directly connected node to the origin node $O$. The links in this set $L_{1}$ will be considered for determination of the minimum weight.

$L_{2}=$ is the set of links, which will never participate in the shortest path. Initially this is an empty set. The links in this set will be a collection of those links that exist between any two labeled nodes with positive weight. The positive weight indicates that travel along the link with positive weight between two labeled nodes will only result in an inferior route. The elements in this set can be one way or two way links.

$L_{3}^{(O, k)}=$ is a set of links that give rise to the shortest path between the nodes $O$ and $k$. This path gets unfolded with the help of the second element $j$ of the label.

\section{Definition 3. Minimum weight}

Since a label on the source node can be easily created, the set $L_{0}$ will not be be an empty set. Thus the set $L_{1}$ will also not be an empty set. Hence minimum weight will always exist, which may not be unique.

\section{Definition 4. Modified weight}

Once a minimum weight has been obtained and a node $k$ has been labeled, it will result in modified weights of all links joining the node $k$ to other nodes of the network. Modified weight associated with the link $(k, j)$ reflects the total distance from the origin node $O$ to the node $j$ vis the node $k$. The modified weight with links $(k, r)$ will represent total distance from the origin node $O$ to the node $r$ and the total distance from the origin node $O$ to the node $k$ will be zero. 
The following observations are also necessary for development of the algorithm.

\section{Observation 1}

The origin node $O$ is the start node. The shortest path and the corresponding distance is required from the node $O$ to the destination node $D$ and the first link on the required path will always be one of the links that directly joins the origin node $O$ to some other node $j$ of the given network in the direction $(O \rightarrow j)$.

\section{Observation 2}

Since all links are non-directed, theoretically one can return to the origin node $O$ from any other node $j$ which is directly connected with node $O$ but such a path from any node $j$ to the node $O$ will only increase the total distance between the nodes $O$ and the node $D$. Thus such a revisit to node $O$ from any node $j$ will not be appropriate with regards to the shortest path. Proof: Let the length of the link $(j, O)$ be denoted by $d_{(j, O)}>0$ which is a positive quantity. Let the shortest path distance between the nodes $(O, D)$ be denoted by $s d_{(O, D)}$. The length of the path from the node $j$ via the node $O$ to the destination will be given by $\left(d_{j, O)}+s d_{(O, D)}\right.$ which will be longer compared to the distance $s d_{(O, D)}$, which by definition is the shortest distance.

\section{Observation 3}

If a node $k$ has been labeled, the shortest path and the shortest distance from the origin node $O$ to the labeled node $k$ is known. As from Observation 2 , it is clear that returning to a labeled node from any unlabeled node can only increase the distance. Thus for purposes of the shortest path all nondirected links from the labeled node $k$ to unlabeled nodes $i$ will be operating as directed links $(k \rightarrow i)$, where node $k$ is a labeled node and node $i$ is a unlabeled node.

\section{Observation 4}

Since initially the origin node $O$ is a labeled node, the links contained in the set $\left\{L_{1}\right\}$ are the directly connected links originating from the node $O$ to all other nodes. These links may be temporarily treated as directed links from $O$ to those nodes $j$. Thus the minimum weight of the links in the set $L_{1}$ can be easily determined. Suppose the minimum weight is associated with the link $(O \rightarrow k)$. If this minimum weight is subtracted from this minimum weight link, the altered associated weight will be 0 on link $(O \rightarrow k)$ and if the same minimum weight is added to all links from the node $k$ in the 
direction $(k \rightarrow j)$, the new weight in the direction $(k \rightarrow O)$ will be twice the original weight. Note that one more node has been labeled and this is one iteration of the labeling process.

\section{Observation 5}

Since in each iteration of the labeling process, one more unlabeled node is labeled, the process must terminate in at most $(|M|-1)$ iterations. Alternatively, as soon as the the destination node is labeled and become a member of the set $L_{0}$, the process terminates.

\section{The minimum weight algorithm for the non- directed network}

The minimum weight algorithm consists of the following steps: Step 1: Label the origin node $O$ as $O_{(0, O, 0)}$ and the set $\left\{L_{1}\right\}$ will be comprised of links from the node $O$ to nodes $j$, which are directly connected. These links will be temporarily treated as directed links from the node $O$ to the node $j$ for determination of the minimum weight. The set $L_{2}=\{\phi\}$ is an empty set.

Find the minimum weight associated with a link that belongs to the set $\left\{L_{1}\right\}$. Let the corresponding node associated with the minimum weight be denoted by node $j$ and the minimum weight be denoted by $w_{(O, j)}$.

Now label the node $j$ that corresponds to the minimum weight as $j_{\left(1, O, w_{(O . j)}\right)}$. The number 1 in the label on node $j$ indicates that it is the first labeled node after the initial labeled node $O$. The minimum distance to this node $j$ from the origin node $O$ is $w_{(O, j)}$. The shortest path from the origin node $O$ to the node $j$ is formed by the link $(O, j)$. All other links from the node $j$ will have different weights in two directions as the minimum weight will have to be added in the direction $(j \rightarrow k)$. Go to step 2 .

Step 2: Based on the weight value $w_{(O, j)}$, modify link weights as follows:

1. The new weight associated with the link $(O, j)$ will be $w_{(O, j)}-w_{(O, j)}=0$.

2 . New weights associated with all directly connected links $(j, k)$ will be given by $w_{(j, k)}+w_{(O, j)}$. Thus the weight on the link in the direction $(j \rightarrow O)$ will be twice the original weight.

3. New weights of all the remaining links not connected with the node $j$ will remain unaltered.

4. Upgrade the sets $L_{0}, L_{1}$ and $L_{2}$.

Step 3: Now the nodes $O$ and $j$ are labeled nodes. Using the Observation 3, one can once again temporarily assume all undirected links connected with the nodes $O$ and $j$ as directed links going away from them. Once again find the minimum weight associated with the directed links and label one more node as the new labeled node. 
Step 4: Is the node $D$ labeled? If yes, stop and the shortest path has been determined. If not, go to step 2.

\section{Why at the end of the process, one finds the shortest path?}

Note that at each iteration, total distance of a path joining the origin node $O$ to the destination node $D$ remains unaltered and all associated link distances are non-negative quantities. Since the sum of the modified distances from the origin node $O$ to the destination node $D$ will be comprised of zero total distance, it will constitute the shortest path.

\section{$5 \quad$ Numerical illustration}

We consider a non-directed 6 node network shown in Table 1. The network diagram is shown in Figure 1. Here the node $O$ is the origin node, the node $D$ is the destination node and the nodes $2,3,4$ and 5 are the intermediate nodes.

Table 1: Link distances

\begin{tabular}{|c||c||c||c||c||c||c|}
\hline Nodes & $O$ & 2 & 3 & 4 & 5 & $D$ \\
\hline$O$ & - & 6 & 12 & $\infty$ & $\infty$ & $\infty$ \\
2 & 6 & - & 5 & 10 & 8 & $\infty$ \\
3 & 12 & 5 & - & 3 & 2 & $\infty$ \\
4 & $\infty$ & 10 & 3 & - & 7 & 9 \\
5 & $\infty$ & 8 & 2 & 7 & - & 11 \\
$D$ & $\infty$ & $\infty$ & $\infty$ & 9 & 11 & - \\
\hline
\end{tabular}




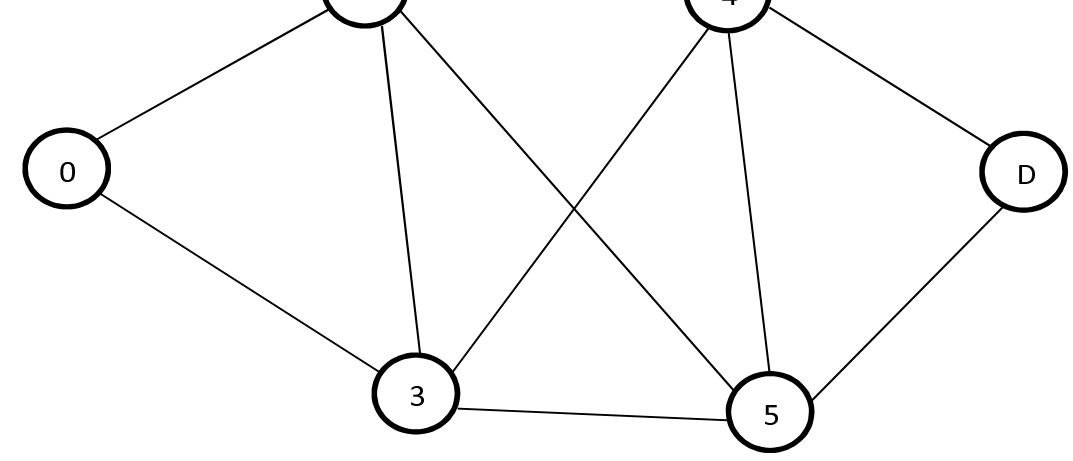

Figure 1: Network diagram of numerical example.

Since the non-directed links $(O, j)$ will be used only in the direction $(O \rightarrow j)$, we indicated in Table 2 , the direction for each entry. Initially the

Table 2: Modified link distances

\begin{tabular}{|c||c||c||c||c||c||c|}
\hline Nodes & $O$ & 2 & 3 & 4 & 5 & $D$ \\
\hline$O_{(0, O .0)}$ & - & $6_{(O, 2)}$ & $12_{(O, 3)}$ & $\infty$ & $\infty$ & $\infty$ \\
2 & $6_{(2, O)}$ & - & $5_{(2,3)}$ & $1_{(2,4)}$ & $8_{(2,5)}$ & $\infty$ \\
3 & $12_{(3, O)}$ & $5_{(3,2)}$ & - & $3_{(3,4)}$ & $2_{(3,5)}$ & $\infty$ \\
4 & $\infty$ & $10_{(4,2)}$ & $3_{(4,3)}$ & - & $7_{(4,5)}$ & $9_{(4, D)}$ \\
5 & $\infty$ & $8_{(5,2)}$ & $2_{(5,3)}$ & $7_{(5,4)}$ & - & $11_{(5, D)}$ \\
$D$ & $\infty$ & $\infty$ & $\infty$ & $9_{(D, 4)}$ & $11_{(D, 5)}$ & - \\
\hline
\end{tabular}

sets $L_{0}=\{O\}, L_{1}=\{(O \rightarrow 2),(O \rightarrow 3)\}$ and $L_{2}=\{\phi\}$. The minimum weight $=$ minimum $(6,12)=6$ corresponds to the link $(O \rightarrow 2)$, which will result in a label at the node 2 . This label also results in the path set $L_{3}^{(O \rightarrow 2)}$ is the link $(O \rightarrow 2)$ with minimum distance $=6$.

The link distances in Table 2 will have to be modified by subtracting the minimum weight $w_{(O, 2)}=6$ from the link $(O \rightarrow 2)$ and added to all links joining the node 2 to all other nodes, i.e. links $\{(2 \rightarrow O),(2 \rightarrow 3),(2 \rightarrow$ $4),(2 \rightarrow 5)\}$. Thus the implementation of the first iteration will result in Table 3. The updated sets are: $L_{0}=\{O, 2\}, L_{1}=\{(O \rightarrow 3),(2 \rightarrow 3),(2 \rightarrow$ $4),(2 \rightarrow 5)\}, L_{2}=\{(2 \rightarrow O)\}$. The minimum weight will be given by $\min (12,11,16,14)=11$, which leads to the label on the node 3 as shown in Table 4 . The path set $L_{3}^{(O \rightarrow 3)}=\{O \rightarrow 2 \rightarrow 3\}$ with minimum distance between the nodes $O$ and 3 is equal to 11 .

The distance 11 will be subtracted from the link $(2 \rightarrow 3)$ and added to links $(3 \rightarrow O),(3 \rightarrow 2),(3 \rightarrow 4)$ and $(3 \rightarrow 5)$, which are shown in Table 4 . From Table 4 , the updated sets are: $L_{0}=\{O, 2,3,\} ; L_{1}=\{(2 \rightarrow 4),(2 \rightarrow$ $5),(3 \rightarrow 4),(3 \rightarrow 5)\}, L_{2}=\{(O, 3),(2 \rightarrow O),(3 \rightarrow 2)\}$. 
Table 3: Modified link distances after iteration 1

\begin{tabular}{|c||c||c||c||c||c||c|}
\hline Nodes & $O$ & 2 & 3 & 4 & 5 & $D$ \\
\hline$O_{(0, O .0)}$ & - & $0_{(O, 2)}$ & $12_{(O, 3)}$ & $\infty$ & $\infty$ & $\infty$ \\
$2_{(1, O, 6)}$ & $12_{(O, 2, O)}$ & - & $11_{(O, 2,3)}$ & $16_{(O, 2,4)}$ & $14_{(O, 2,5)}$ & $\infty$ \\
3 & $12_{(3, O)}$ & $5_{(3,2)}$ & - & $3_{(3,4)}$ & $2_{(3,5)}$ & $\infty$ \\
4 & $\infty$ & $10_{(4,2)}$ & $3_{(4,3)}$ & - & $7_{(4,5)}$ & $9_{(4, D)}$ \\
5 & $\infty$ & $8_{(5,2)}$ & $2_{(5,3)}$ & $7_{(5,4)}$ & - & $11_{(5, D)}$ \\
$D$ & $\infty$ & $\infty$ & $\infty$ & $9_{(D, 4)}$ & $11_{(D, 5)}$ & - \\
\hline
\end{tabular}

Table 4: Modified link distances at the end of iteration 2

\begin{tabular}{|c||c||c||c||c||c||c|}
\hline Nodes & $O$ & 2 & 3 & 4 & 5 & $D$ \\
\hline$O_{(0, O .0)}$ & - & $0_{(O, 2)}$ & $12_{(O, 3)}$ & $\infty$ & $\infty$ & $\infty$ \\
$2_{(1, O, 6)}$ & $12_{(O, 2, O)}$ & - & $0_{(O, 2,3)}$ & $16_{(O, 2,4)}$ & $14_{(O, 2,5)}$ & $\infty$ \\
$3_{(2,2,11)}$ & $23_{(O, 2,3, O)}$ & $16_{(O, 2,3,2)}$ & - & $14_{(O, 2,3,4)}$ & $13_{(O, 2,3,5)}$ & $\infty$ \\
4 & $\infty$ & $10_{(4,2)}$ & $3_{(4,3)}$ & - & $7_{(4,5)}$ & $9_{(4, D)}$ \\
5 & $\infty$ & $8_{(5,2)}$ & $2_{(5,3)}$ & $7_{(5,4)}$ & - & $11_{(5, D)}$ \\
$D$ & $\infty$ & $\infty$ & $\infty$ & $9_{(D, 4)}$ & $11_{(D, 5)}$ & - \\
\hline
\end{tabular}

The minimum weight will be given by $\min (16,14,14,13)=13$. This minimum weight corresponds to the node 5 by using the link $(3 \rightarrow 5)$. Thus 13 will have to be subtracted from the link weight $(3 \rightarrow 5)$ and added to link weights $(5 \rightarrow 2),(5 \rightarrow 3),(5 \rightarrow 4),(5 \rightarrow D)$. This is shown in Table 5 . From Table 5 , the updated sets $L_{0}=\{O, 2,3,5\}, L_{1}=\{(2 \rightarrow 4),(3 \rightarrow 4),(5 \rightarrow$ $4),(5 \rightarrow D)\}, L_{2}=\{(O, 3),(2 \rightarrow O),(3 \rightarrow 2),(5 \rightarrow 2),(5 \rightarrow 3)\}$. The minimum weight will be given by $\min (16,14,20,24)=14$, which corresponds to node 4 . Labeling node 4 and updating the network and the associated weights, we get Table 6 . The minimum distance path from the origin node $O$ to the node 4 is given by $\{O \rightarrow 2 \rightarrow 3 \rightarrow 4$ ) with minimum distance 14 . From Table 6 , the updated sets $\left.L_{0}=\{O, 2,3,4,5\}, L_{1}=\{4 \rightarrow D),(5 \rightarrow D)\right\}$, $L_{2}=\{(O, 3),(2 \rightarrow 4),(4 \rightarrow 2),(5 \rightarrow 2)\}$. The minimum weight will be given by $\min (23,24)=23$, which corresponds to the destination node $D$. Labeling the node $D$ and updating the network and the associated weights, we get Table 7 . 
Table 5: Modified link distances at the end of iteration 3

\begin{tabular}{|c||c||c||c||c||c||c|}
\hline Nodes & $O$ & 2 & 3 & 4 & 5 & $D$ \\
\hline$O_{(0, O .0)}$ & - & $0_{(O, 2)}$ & $12_{(O, 3)}$ & $\infty$ & $\infty$ & $\infty$ \\
$2_{(1, O, 6)}$ & $12_{(O, 2, O)}$ & - & $0_{(O, 2,3)}$ & $16_{(O, 2,4)}$ & $14_{(O, 2,5)}$ & $\infty$ \\
$3_{(2,2,11)}$ & $23_{(O, 2,3, O)}$ & $16_{(O, 2,3, O)}$ & - & $14_{(O, 2,3,4)}$ & $0_{(O, 2,3,5)}$ & $\infty$ \\
4 & $\infty$ & $10_{(4,2)}$ & $3_{(4,3)}$ & - & $7_{(4,5)}$ & $9_{(4, D)}$ \\
$5_{(3,3,13)}$ & $\infty$ & $21_{(O, 2,3,5,2)}$ & $15_{(O, 2,3,5,3)}$ & $20_{(O, 2,3,5,4)}$ & - & $24_{(O, 2,3,5, D)}$ \\
$D$ & $\infty$ & $\infty$ & $\infty$ & $9_{(D, 4)}$ & $11_{(D, 5)}$ & - \\
\hline
\end{tabular}

Table 6: Modified link distances at the end of iteration 4

\begin{tabular}{|c||c||c||c||c||c||c|}
\hline Nodes & $O$ & 2 & 3 & 4 & 5 & $D$ \\
\hline$O_{(0, O .0)}$ & - & $0_{(O, 2)}$ & $12_{(O, 3)}$ & $\infty$ & $\infty$ & $\infty$ \\
$2_{(1, O, 6)}$ & $12_{(O, 2, O)}$ & - & $0_{(O, 2,3)}$ & $16_{(O, 2,4)}$ & $14_{(O, 2,5)}$ & $\infty$ \\
$3_{(2,2,11)}$ & $23_{(O, 2,3, O)}$ & $16_{(O, 2,3,2)}$ & - & $0_{(O, 2,3,4)}$ & $0_{(O, 2,3,5)}$ & $\infty$ \\
$4_{(4,3,14)}$ & $\infty$ & $24_{(O, 2,3,4,2)}$ & $17_{(O, 2,3,4,3)}$ & - & $21_{(O, 2,3,4,5)}$ & $23_{(O, 2,3,4, D)}$ \\
$5_{(3,3,13)}$ & $\infty$ & $21_{(O, 2,3,5,2)}$ & $15_{(O, 2,3,5,3)}$ & $20_{(O, 2,3,5,4)}$ & - & $24_{(O, 2,3,5, D)}$ \\
$D$ & $\infty$ & $\infty$ & $\infty$ & $23_{(O, 2,3,4, D)}$ & $24_{(O, 2,3,5, D)}$ & - \\
\hline
\end{tabular}

Table 8 summarizes the optimal solution. 
Table 7: Modified link distances at the end of iteration 5

\begin{tabular}{|c||c||c||c||c||c||c|}
\hline Nodes & $O$ & 2 & 3 & 4 & 5 & $D$ \\
\hline$O_{(0, O .0)}$ & - & $0_{(O, 2)}$ & $12_{(O, 3)}$ & $\infty$ & $\infty$ & $\infty$ \\
$2_{(1, O, 6)}$ & $12_{(O, 2, O)}$ & - & $0_{(O, 2,3)}$ & $16_{(O, 2,4)}$ & $14_{(O, 2,5)}$ & $\infty$ \\
$3_{(2,2,11)}$ & $23_{(O, 2,3, O)}$ & $16_{(O, 2,3,2)}$ & - & $0_{(O, 2,3,4)}$ & $0_{(O, 2,3,5)}$ & $\infty$ \\
$4_{(4,3,14)}$ & $\infty$ & $24_{(O, 2,3,4,2)}$ & $17_{(O, 2,3,4,3)}$ & - & $21_{(O, 2,3,4,5)}$ & $0_{(O, 2,3,4, D)}$ \\
$5_{(3,3,13)}$ & $\infty$ & $21_{(O, 2,3,5,2)}$ & $15_{(O, 2,3,5,3)}$ & $20_{(O, 2,3,5,4)}$ & - & $24_{(O, 2,3,5, D)}$ \\
$D_{(5,4,23)}$ & $\infty$ & $\infty$ & $\infty$ & $23_{(O, 2,3,4, D)}$ & $24_{(O, 2,3,5, D)}$ & - \\
\hline
\end{tabular}

Table 8: Distance and path from the origin node $O$ to other nodes

\begin{tabular}{|c||c||c|}
\hline From node $O$ to node $j$ & Min Distance & Shortest Path \\
$j=O$ & 0 & $O \rightarrow O$ \\
$j=2$ & 6 & $O \rightarrow 2$ \\
$j=3$ & 11 & $O \rightarrow 2 \rightarrow 3$ \\
$j=4$ & 14 & $O \rightarrow 2 \rightarrow 3 \rightarrow 4$ \\
$j=5$ & 13 & $O \rightarrow 2 \rightarrow 3 \rightarrow 5$ \\
$j=D$ & 23 & $O \rightarrow 2 \rightarrow 3 \rightarrow 4 \rightarrow D$ \\
\hline
\end{tabular}

\section{Conclusion}

The major contribution of this paper is the proposed new minimum weight labelling method for determining a shortest route in a non-directed network. The key feature of this method is that for an $m$ node network the algorithm developed finds an optimal solution in at most $(m-1)$ iterations. As is the case with any new methodology there are some areas for possible extension and improvement. For instance a rigorous proof of the complexity of the algorithm is needed. Another area is development of appropriate software for the proposed algorithm and testing it on larger problems and carrying out a comparative analysis with other existing methods. Munapo (2010) extended the minimum weight label approach on project activity analysis without drawing out a network diagram, which may require use of dummy activities. The approach discussed in this paper deserve similar considerations. These areas will be studied elsewhere. 


\section{Acknowledgments}

The authors are grateful to the numerous people for helpful comments on this paper.

\section{References}

[1] Banasal SP \& Kumar S, 1977, Maximum Reliability Route subject to M improvements in a directed Graph, IEEE Transactions on Reliability,R26(2), pp. 103-05.

[2] Dantzig GB, 1960, On the shortest route through a network, Management Sci., 6(2), pp. 187-190.

[3] Dijkstra EW, 1959, A note on two problems in connection with graphs, Numerische Mathematick,1, pp. 269-271.

[4] Floyd RW, 1962, Algorithm 97: Shortest path, Communications of ACM, 5(6), pp. 345.

[5] Ford LR, 1956, Network flow theory, Report P-923, The Rand Corporation.

[6] Kumar S, Arora H \& Bappoo R, 1999, Kth best solution of a system, Journal of Applied Cybernetics, SCIMA, 26(1), pp. 3-19.

[7] Munapo E, 2010, Project Activity Analysis without the network model, South African Journal of Industrial Engineering, 21(1), pp. 81-92.

[8] Munapo E, Jones BC \& Kumar S, 2008, Minimum incoming weight label method and its application in CPM networks, ORiON, 24(1), pp. $37-48$.

[9] Zhan FB, 1997, Three shortest path algorithms on real road networks: Data structures and procedures, Journal of Geographic Information and Decision Analysis, 1(1), pp. 69-82.

[10] Zhan FB\& Noon CE, 1998, Shortest Path Algorithms: An evaluation using real road networks, Transportation Science, 32(1),pp. 65-73. 


\section{University Library}

\section{- M M N E R VA A gateway to Melbourne's research publications}

Minerva Access is the Institutional Repository of The University of Melbourne

\section{Author/s:}

Kumar, S;Munapo, E;Ncube, O;Sigauke, C;Nyamugure, P

Title:

A minimum weight labelling method for determination of a shortest route in a non-directed network

\section{Date:}

2013-01-01

\section{Citation:}

Kumar, S., Munapo, E., Ncube, O., Sigauke, C. \& Nyamugure, P. (2013). A minimum weight labelling method for determination of a shortest route in a non-directed network. International Journal of System Assurance Engineering and Management, 4 (1), pp.13-18. https://doi.org/10.1007/s13198-012-0140-7.

Persistent Link:

http://hdl.handle.net/11343/283307 